\title{
The Hidden Benefits of a CALL Facility:
}

\author{
Alida Abbott \\ University of Victoria
}

The objective of this research was to conduct a small scale 'interpretive qualitative and quantitative' project in order to investigate the unseen advantages of a CALL Facility. The principal goal was to determine how the sociocultural context of a computer mediated environment affects the learning experience of its users. By sociocultural it is meant: the prior language learning experience of students, their attitudes and motivation towards learning another language, their cultural and linguistic background, exposure to computers and how these factors interact with one another and have an effect on a computer mediated environment. More specifically, this research has centered on the backgrounds and attitudes of the learners and why they utilize this facility, and what it is that motivates and encourages them to persist in using this learning space. This research is concerned with an area, which has received little attention, one that concentrates on the attitudes towards a computer-enhanced environment by both international and domestic students studying foreign languages in the CALL Facility at the University of Victoria.

This project made use of the CALL Facility at the University of Victoria as a case study and a site for investigation in order to better understand and highlight the workings of a computer-enhanced language-learning environment. An online questionnaire, which elicited qualitative and quantitative data, paired with quantitative statistical data retrieved from Wicket (the facility's central database program used to track student data) were used as the methods of data collection in order to achieve a complete and thorough understanding and perception of the connection between a CALL environment and the learning practice of its users.

Background / Context
CALL is a rapidly evolving academic field, which is increasingly exploring the role of information and communication technologies in language learning and pedagogy. Due to the diversity and changeability of the field, which includes the emergence of new theoretical, methodological, and learning paradigms, this field has become even more dynamic in the last decade (Chapelle 2001). Although considerable research has been conducted to attempt to 
understand the link between computers and language learning and how to implement technology successfully in language programs, (Beatty 2003; Cameron 1999; Chapelle 2001; Chapelle 1997; Fotos 2004; Gillespie 2000: Liddell 1997; Warschauer 2002, 2000, 1997, 1996; Warschauer \& Healey 1998) relatively little is known about how a computer mediated environment affects the learning process of its users. Interestingly, most computer-assisted language learning itself takes place in a language lab (Fotos 2004), yet very little research has been dedicated to the findings on the interaction between the learner, (including their cultural and linguistic background) and the computer mediated environment itself. One of the most recent studies has examined the importance of a comfortable atmosphere and good support staff, but does not focus on the effects of the environment and student response (Fotos 2004). Other research has begun to concentrate on the emergence and popularity of "the sociopsychological and cultural realities of the virtual community" (AbdelnourNocera 1998, 193); however, neglects to focus on the importance of a real-life environment. According to my research, nothing has targeted the discussion of the relationship between the sociocultural contexts of a CALL facility and how this affects the learning process of its users. Nor has any research targeted the attitudes of language learners towardsa technology-enhanced language-learning environment. The intention of this research is to fill this gap in order to better understand the interrelation between learners' backgrounds, expectations, needs and the language learning process in a CALL Facility.

Literature Review The field of CALL has a rich history dating back to the early 1950's (Beatty 2003). Early CALL applications were very simple and linear following 'drill and kill' applications. During the 1970's and 1980's, CALL applications progressed from linear programs to more diverse ways of exploring imitations of reality and use of the imagination (Beatty 2003). The progression of highly interactive computer based programs with the utilization of sophisticated programming is now allowing users to do a significant amount of functions. Nowadays, there is more of a focus on web-based applications, and more resources are being transferred from analogue to digital format. Clearly, computers are used for a variety of reasons; however, the role that CALL has played and can play in second and foreign language teaching and learning has become essential. There is much literature available surrounding the topic of CALL and the types of CALL based tasks that can be beneficial to language learning (Beatty 2003; Cameron 1999; Chapelle 2001; Liddell 1997; Fotos 2004; Warschauer 1996,1997,2002; Warschauer \& Healy 1998). 
The benefits of computers in language learning are numerous; factors such as the Internet, ease of networking capabilities, multilingual accessibilities, multicultural educational opportunities, and the mere fact that these machinescan lessen the 'fear factor' associated with learning another language are all elements, which encourage their use. Students can use these machines to chat with other individuals in a variety of languages in another country, they can listen to news broadcasts in a multitude of languages; learn colloquial slang and idiomatic expressions through various websites. However, therearenegative factors that are also present and cannot be left out; although computers have incredible capabilities to simulate real life situations, they are machines and as such they tend to diminish the socially interactive aspects involved in language learning. Not everyone is able to appreciate the full potential of computers; technological limitations paired with social and economic realities unfortunately do not allow for everyone to benefit from the wealth of capabilities that these machines can offer. Computers as instruments have been extremely helpful in assisting and augmenting the transmission of knowledge across the globe; however, even with sophisticated software and programming, human interaction is still an essential quality.

Ideal CALL facilities can help bridge the gap between the negative and the positive factors and allow for real life interaction, while promoting the social and cultural aspects of language learning, which are essential ingredients. CALL facilities have helped students in many ways become familiar with language, culture and technology. Computer based education has seen many pedagogical shifts over the past decades. Two of the most recent changes have witnessed a shift from earlier models of computerbased instruction; a communicative CALL and integrative CALL based approach (Warschauer 2000). The differences lie in that the communicative approach stressed communication for the sake of communication, not relying heavily on content, whereas the integrative approach emphasized a socio-cognitive type of methodology, where both the content and nature of the conversations were very vital components (Warschauer 2000). The sociocultural context and environment of CALL Facilities is an area, which has received little attention. Existing literature comments largely on the aspects of incorporating it within learning activities, and how technology itself can bridge the gap between culture and language; however, the social dynamics of language learning within a technology enhanced environment have largely been left alone. Much of the current literature pertaining to sociocultural theory refers back to Vygotskian views that place an emphasis on the social world where learning and literacyemerge 
(Pérez 1998, Warschauer 1997). In essence, the evolution of CALL interms of sociocultural literacy practice can be nicelysummed up as follows: "The early days encompassed transmission (passive instruction), which led to the phase of constructivism (more autonomy and choices for learners) and now these have been superseded by dialogism, where meaning should come from and is developed in interaction between the individual and social audience" (Warschauer 1997, 92).

Motivation, or the desire to learn another language is a complex construct, difficult to define, problematic to measure, and challenging to link directly to the success in second language learning. Language learning itself is a unique process and involves different attitudes and behaviors, which are linked to a variety of factors, which, in turn, largely determine the success or failure of the language learner. Thereiscertainly no one pattern of motivation that a learner adopts in order to achieve a certain goal, but, with education and awareness both learners and educators can be conscious of some of the socio-cultural and psychosocial factors that contribute to the language learning experience. These factors, combined with appropriate learning strategies, can assist a learner in the development and progression of the L2 learning experience. While past research has largely focused on the social psychological factors and theories of language learning, more recently, research has focused and explored different dimensions of motivation, stemmingfrom theories in educational researchand the psychology of learning (Dörnyei 2003). Understanding the influential factors, which motivate learners to acquire an L2, has a profound impact on how the motion of motivation in education is viewed. Acknowledging and validating these important social, cultural and contextual factors in the language learner can assist in making the experience valuable, productive and more successful.

As there is a lack of concrete research available pertaining to the effects of a CALL environment on the learning practice of its users, additional research is required in order to be able to better understand if and how a CALL environment does indeed have an effect on the learning practice of its users. It is hoped that this research will provide some solid insights into the relationship between a CALLenvironment and effective language learning. *

Alida Abbott holds an MA in Applied Linguistics from YorkUniversity with a focus in second languageacquisition and CALL. Hermain research interests include pedagogical uses of CALL and computer assisted research in foreign language acquisition. She is the Coordinator of the CALL Facility at the University of Victoria. 